\title{
Preface to the special issue on "Demographic and temporal heterogeneities in infectious disease epidemiology"
}

\author{
B. Buonomo ${ }^{1}$ - N. Chitnis ${ }^{2,4}$ - A. d'Onofrio ${ }^{3}$
}

Received: 10 August 2017 / Revised: 1 September 2017 / Published online: 7 February 2018 (C) Università degli Studi di Napoli "Federico II" 2018

Mathematical models of the spread and control of infectious diseases in humans should not simply be mathematically interesting. They ought also to provide some qualitative or quantitative information that can in some way be of interest for public health. In many cases, even simple mathematical models have been able to fulfill the difficult task of providing help to public health scientists to better understand the interplay/interdependence between the dynamics of the disease and the control measures to be enacted. Four pillars of public health campaigns for controlling the spread of infectious diseases are: treating infectious humans; vaccination to prevent infection; reduction of risky behaviors; and vector control, in the case of vector borne diseases. All these pillars depend on various factors of heterogeneity that must be taken into account if we want to pass from a vague utility to some more robust role for mathematical epidemiology. The first heterogeneity factor is the age structure of population. First and foremost, the risk of disease-induced mortality for many diseases is age dependent, with children and elderly with a higher risk of fatality. The pattern of contacts is also strongly

$凶$ B. Buonomo

buonomo@unina.it

N. Chitnis

nakul.chitnis@unibas.ch

A. d'Onofrio

alberto.donofrio@i-pri.org

1 Department of Mathematics and Applications, University of Naples Federico II, via Cintia 80126, Naples, Italy

2 Department of Public Health and Epidemiology, Swiss Tropical and Public Health Institute, Socinstrasse 57, Postfach, 4002 Basel, Switzerland

3 International Prevention Research Institute, 95 Cours Lafayette, 69006 Lyon, France

4 University of Basel, Basel, Switzerland 
influenced by the age of the subjects, as we all very well know from our daily life experience. On the other hand, the age at which a subject is vaccinated strongly affects the effectiveness of the vaccine. Therefore, determining the optimal age(s) to deliver the vaccine dose(s) is crucial to ensure the effectiveness of a vaccination campaign. Another important factor is the waning efficacy of some vaccines which implies that persons vaccinated in their childhood have to be re-vaccinated as adults. Practical problems are enormous and most often unknown to many mathematical modelers. Delivering vaccines to children is challenging due to the pseudo-rational opposition to vaccination by their parents [1,2]. Moreover, making adults and elderly people aware of the necessity to have annual flu vaccinations or re-calls of their childhood vaccine is also extremely challenging [3].

The above-mentioned phenomena of opposition/hesitancy to vaccination is typical of the post-trust society in which we are living [4] and where mandatory vaccination are increasingly often avoided. This opposition/hesitancy induces a new kind of heterogeneity, which is represented by time varying vaccination rates. The study of this heterogeneity and of other human-behavior dependent phenomena in the context of the spread and control of infectious diseases form the basis of a new highly interdisciplinary scientific field: the behavioral epidemiology of infectious diseases [2].

For many diseases the pattern of contacts and the probability of being infected during a contact with an infectious subject follow a strong seasonal pattern. Indeed, we recall that: (i) the pattern of contacts is influenced by social phenomena, such as the school calendar for childhood diseases and alternating periods between work and holidays for adults or even, in the past, recurrent events with high levels of crowding, such as large fairs; (ii) the contagion probability per contact may be affected by temperature and humidity, which has a periodic pattern. The risk of infection for vector-borne diseases often varies periodically due to seasonality in rainfall and temperature. During the rainy season, water collects in containers, such as flower pots and tyres, or puddles where mosquitoes and other vectors can breed. This increase in breeding sites leads to increases in vector densities during the rainy season. Insect vectors are ectotherms so physiological processes of the vector and pathogens in the vector depend on the ambient temperature. At warmer temperatures, pathogens develop faster in vectors, and vectors bite more frequently but may conversely suffer higher mortality rates (especially if humidity is low).

The modeling implication of these periodic biological and social phenomena is that the resulting force of infection is non-autonomous, i.e. it exhibits a temporal heterogeneity source different from the dynamics of the epidemic state variable.

The public health consequence of these temporal heterogeneities is that the prevalence of some infectious diseases has strong seasonality, regular or-most often-irregular. This induces many practical problems both for health-care and for prevention campaigns. Thus the inherent age-structure of the populations, behavioral heterogeneities, time-structure of the force of infection and, for the vector-borne diseases, the seasonality in vector densities and contact rates remarkably affect the strategies that public health authorities can develop to control epidemics/pandemics. This influence acts on multiple fronts: from the design of optimal strategies of inter- 
ventions to the correct scheduling of beds in the infectious diseases and emergency departments of hospitals.

The same relevance is intrinsic for an optimal implementation of awareness campaigns aimed at modifying risky behaviors, especially during large epidemics, and to increase vaccination rates. Acquiring a clear idea of the dynamics of epidemics and of their inherent causes is of increasing relevance in a world where economic resources devoted to public health are limited and in many cases decreasing.

Unfortunately, in the undergraduate and even in the graduate study in mathematical biology, formal courses in public health sciences and practice are seldom included in the curricula of study. Thus the relevance of the above-mentioned issues may not be well perceived. As a consequence, heterogeneity factors are neglected even when modeling diseases strongly impacted by them.

With this special issue of Ricerche di Matematica we have a double aim. The first is to urge more theoretically oriented colleagues to fully understand the public health relevance of the study of epidemic models including heterogeneities. The second is to illustrate to the wide mathematical world, the 'mathematical beauty' of such models. Indeed, the inclusion of the above-illustrated heterogeneities leads to elegant models whose mathematical analysis is most often challenging. As a general rule, the closer a mathematical model is to reality the harder is its mathematical analysis.

The first article of this Special Issue is by Buonomo, Chitnis and d'Onofrio, who review historical and current mathematical epidemiology literature on the temporal heterogeneity of the contact rate.

The second paper, authored by Billings and Forgoston, is on the role of stochastic forcing due to heterogeneity in the contact rate of epidemic stochastic models. The focus is on semi-analytical methods of statistical physics and on noise-induced rare events, such as the spontaneous disease extinction. Quasi-stationary solutions and the optimal path to extinction are identified.

Multi-group diseases are strongly characterized by a group-dependent heterogeneity. This is the focus of the paper by Lunelli and Pugliese, who propose and investigate a multi-group model (both in its deterministic and stochastic version) with two level of mixing: intra-group and on the whole population. The authors show that the contact rate heterogeneity can affect the attack ratio.

The next two papers focus on tuberculosis. A first paper, by Esteva and IbargüenMondragón, is a within-host model of the onset of antibiotic resistance in Mycobacterium tuberculosis. Onset of antibiotic resistance is an emergent topic of the utmost relevance in global public health. The second paper by Rocha, Silva and Torres addresses another emergent problem of increasing relevance: the public health effects of immigration from countries where the prevalence of tuberculosis is high. Their model has the interesting feature that its basic reproduction number does not linearly depend on the disease contact rate.

The next paper, by Chaves, estimates the basic reproduction number from certain age-structured models where the contact rate is constant and age-independent.

The paper by Röst and colleagues focuses on the development and global analysis of a model of spread and treatment of influenza, where treatment depends on the age of the infection. Indeed, in influenza the promptness of therapy after the onset of symptoms is correlated with the success of the treatment. 
In mosquito-borne diseases, an effective form of control of vectors is the release of sterile mosquitoes. This is the subject of the contribution by Yang Li and Jia Li, who propose and analyze a discrete-time model of a population of mosquitoes in presence of time-varying release of sterile mosquitoes. The paper by Sánchez et al. also focuses on vector-borne diseases. In this article, the authors compare outbreaks in Costa Rica of two very serious Aedes aegypti-borne diseases: dengue and chikunguya. These two diseases exhibit similar symptoms, thus leading to an over-reporting of dengue and to an under-reporting of chikunguya. Therefore, the authors introduce a single-outbreak model including a class of undiagnosed subjects, which is fit to field data.

Then four papers on behavioral epidemiology follow. In the first, J. Eckalbar and W. Eckalbar assess the impact of seasonality in contact rates in a behavioral model. The synergies between the seasonality in the contact rate and the delayed response of vaccination propensity to information on disease prevalence are analyzed in depth. Buonomo, Chitnis and d'Onofrio in the second of these papers, mathematically investigate the synergy between contact rate heterogeneity and temporal heterogeneity in the variability of the effort of a public health system implementing a vaccine awareness program aimed to increase the vaccine propensity in citizens. The awareness campaign is modeled as an imitation game with periodically varying public health system efforts. Game theory and heterogeneity of the vaccination rates of the newborn babies are also central in the third behavioral epidemiology paper by Hubert and Turinici. In this work, the authors model evolution of each individual as a Markov chain, where the individual vaccination decision optimizes a criterion depending on the time-dependent aggregate (societal) vaccination rate and the future epidemic dynamics. The existence of a Nash mean field game equilibrium is shown. The fourth paper, by Laguzet, assumes as a starting point a mean field epidemic game, and applies advanced Runge-Kutta methods to infer the Nash equilibrium.

The final paper, by Marinoschi, focuses on an area up to now rarely explored in mathematical epidemiology: abstract spatial epidemic models in form of differential inclusions. The inclusion is due to local spatial heterogeneities in the nonlinear diffusive terms consisting in their possible multivalued nature.

Acknowledgements The guest editors fully acknowledge the editor-in-chief, Prof. S. Rionero, for providing them with the great opportunity of preparing this special issue. This opportunity is even more precious because it falls in the year of Mathematical Biology 2018.

\section{References}

1. Buonomo, B., d'Onofrio, A., Lacitignola, D.: Modeling of pseudo-rational exemption to vaccination for SEIR diseases. J. Math. Anal. Appl. 404(2), 385-398 (2013)

2. Manfredi, P., d'Onofrio, A. (eds.): Modeling the interplay between human behavior and the spread of infectious diseases. Springer, New York (2013)

3. EU. Action plan on science in society related issues in epidemics and total pandemics. www.asset-scienceinsociety.eu. Accessed Sept 2017

4. Löfstedt, R.: Risk Management in Post-trust Societies. Palgrave Macmillan, New York (2005) 